TecnoHumanismo. Revista Científica

Setiembre - Noviembre 2021

Volumen 1 / No.3

ISSN: $2710-2394$

pp. $246-265$

https://doi.org/10.53673/th.v1i10.96

https://tecnohumanismo.online

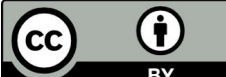

\title{
Predictibilidad de la precipitación en la cuenca alta del mantaro basadas en radiación de onda larga emergente
}

Predictability of precipitation in the upper Mantaro Basin based on emerging longwave radiation

Previsibilidade da precipitação na bacia superior de Mantaro com base na radiação de onda longa emergente

\section{ARTÍCULO GENERAL}

Kenyi Glicerio Cavalcanti Cárdenas

https://orcid.org/0000-0001-9410-3577

kcavalcanti@lamolina.edu.pe

Universidad Nacional Agraria La Molina

Lima - Perú

\author{
Víctor Filiberto Aguilar Vidangos \\ https://orcid.org/0000-0003-2317-4647 \\ vaguilar@lamolina.edu.pe \\ Universidad Nacional Agraria La Molina \\ Lima - Perú
}

Recibido 07 de Abril 2021 | Arbitrado y aceptado 19 de Junio 2021 | Publicado en 03 Setiembre 2021

\section{RESUMEN}

Eventos extremos como El Niño y La Niña modifican la variabilidad hidrológica en muchos lugares del planeta, generando impactos significativos en el bienestar socio económico del país; empero, los procesos físicos y dinámicas atmosféricas vinculados a la generación $y$ al desarrollo de precipitaciones extremas, están aun pobremente estudiados. En la presente investigación, se analiza la variabilidad temporal y espacial de la precipitación, a escala interanual y diaria, durante eventos La Niña, de magnitud moderada y fuerte, identificados durante el período 1998-2015, en la cuenca alta del río Mantaro. Los resultados muestran patrones diarios de precipitación mediante la aplicación de la técnica del Análisis de Componentes Principales, para los meses de mayor precipitación, en años identificados con eventos La Niña. Finalmente, se analizó la capacidad de predictibilidad de la precipitación durante eventos La Niña mediante la utilización de un índice que utiliza valores de OLR medidos en el Pacífico Central (Niño 4) así como en el análisis de los procesos de circulación atmosférica que favorecen la ocurrencia de precipitaciones extremas en la cuenca alta del Mantaro.

Palabras clave: Regionalización de la precipitación, índices de precipitación, patrones diarios de precipitación, cuenca alta del Mantaro, evento La Niña.

\section{ABSTRACT}

Extreme events such as El Niño and La Niña modify the hydrological variability in many parts of the planet, generating significant impacts on the country's socio-economic well-being; however, the physical processes and atmospheric dynamics linked to the generation and development of extreme precipitation are still poorly studied. In the present investigation, the temporal and spatial variability of precipitation is analyzed, on an interannual and daily scale, during La Niña events, of moderate and strong magnitude, identified during the period 1998-2015, in the upper basin of the Mantaro River. The results show daily patterns of precipitation through the application of the Principal Component Analysis technique, for the months of greatest precipitation, in years identified with La Niña events. Finally, the predictability of precipitation during La Niña events was analyzed through the use of an index that uses OLR values measured in the Central Pacific (Niño 4) as well as in the analysis of the atmospheric circulation processes that favor the occurrence of extreme rainfall in the upper Mantaro basin. Keywords: Precipitation regionalization, precipitation indices, daily precipitation patterns, Mantaro high basin, La Niña event.

\section{RESUMO}

Eventos extremos como El Niño e La Niña modificam a variabilidade hidrológica em muitas partes do planeta, gerando impactos significativos no bem-estar socioeconômico do país; entretanto, os processos físicos e a dinâmica atmosférica ligados à geração e desenvolvimento de precipitações extremas ainda são pouco estudados. $\mathrm{Na}$ presente investigação, analisa-se a variabilidade temporal e espacial da precipitação, em escala interanual e diária, durante eventos de La Niña, de magnitude moderada e forte, identificados durante o período 1998-2015, na bacia superior do rio Mantaro. Os resultados mostram padrões diários de precipitação através da aplicação da técnica de Análise de Componentes Principais, para os meses de maior precipitação, em anos identificados com eventos de La Niña. Por fim, a previsibilidade da precipitação durante eventos de La Niña foi analisada através da utilização de um índice que utiliza valores de ROL medidos no Pacífico Central (Niño 4), bem como na análise dos processos de circulação atmosférica que favorecem a ocorrência de eventos extremos. chuvas na bacia do alto Mantaro. Palavras-chave: Regionalização da precipitação, índices de precipitação, padrões diários de precipitação, bacia alta do Mantaro, evento La Niña. 


\section{Introducción}

Uno de los grandes desafíos para la comunidad científica actual, es poder proporcionar información útil y oportuna a los tomadores de decisiones y así poder reducir la vulnerabilidad ante el cambio climático. Si bien los pronósticos probabilísticos de cantidades medias estacionales han mostrado gran utilidad, éstos no responden las grandes interrogantes relacionadas con características específicas de la precipitación, los cuales son usados para pronósticos detallados (e.g., para determinar el inicio y término de una temporada de lluvias en algunos lugares específicos o para el análisis de riesgo de fenómenos meteorológicos de alto impacto). Por otro lado, nuestro conocimiento actual del sistema climático sugiere que aún no es posible satisfacer pronósticos, con un adecuado grado de confiabilidad a muy corto plazo. Asimismo, en los últimos años se ha realizado importantes esfuerzos orientados a entender y caracterizar la variabilidad de la precipitación. Por ejemplo, (Silva et al., 2008) documentaron una tendencia negativa de dos por ciento por década para las precipitaciones y cuatro por ciento por década para periodos de lluvia desde la segunda mitad de 1980 en la cuenca del río Mantaro. Estudios de (Segura et al., 2016) sugieren la presencia de una variabilidad de baja frecuencia en la hidroclimatología de los Andes centrales, posiblemente relacionada a la variabilidad decadal e interdecadal del Pacifico Central- Occidental y de los vientos zonales a $200 \mathrm{hPa}$. Por otro lado, (Sulca et al., 2016) han estudiado los procesos de teleconección entre los Andes centrales y el noreste del Brasil durante eventos de precipitaciones extremas. Si bien estos estudios, muestran un importante avance en el estado del conocimiento relacionado a la ocurrencia de precipitaciones sobre los Andes Centrales, pocos estudios han analizado la posible correlación entre la variabilidad de precipitaciones y los valores de Radiación de Onda Larga Emergente, así como la capacidad de predicción de precipitaciones sobre la base de dicha correlación(Chiodi \& Harrison, 2015).

Por otro lado, si bien la Radiación de Onda Larga Emergente (OLR, por sus siglas en inglés), la Temperatura Superficial del Mar (TSM), Presión a Nivel del Mar (SLP, por sus siglas en inglés) son medidas del estado del sistema acoplado ENSO, los valores del OLR muestran una mayor conexión con anomalías en la atmósfera; generando, en algunos casos, condiciones adecuadas para la ocurrencia de precipitaciones extremas en muchas partes del mundo (Chiodi \& Harrison, 2015). Actualmente, valores de Radiación de Onda Larga Emergente (OLR) se han venido utilizando ampliamente en diversos estudios meteorológicos como un componente del balance de radiación en la 
atmósfera; así como, para colegir cambios en la cantidad y en la altura de las nubes.

Dichos estudios han demostrado que, en los trópicos, los valores de temperatura superficial del mar varían de manera ligera a lo largo de un ciclo anual; por tanto, la presencia de fuertes anomalías en los valores de OLR son un indicador de la presencia de nubes y de la profundidad de estas. Dicha conexión física directa, ha hecho posible la utilización de los valores de OLR para la estimación de la precipitación desde la década de 1980. Por otro lado, si bien existen modelos atmosféricos para la estimación de la variación de calor en la atmósfera, no está claro el grado de corrección de dichos valores, sobre todo en años identificados con la presencia de eventos extremos. Por otra parte, no se cuenta con mediciones directas disponibles de las anomalías del calor en la atmósfera. Por ello, un análisis del comportamiento de OLR en el Pacífico Central puede brindar un mejor entendimiento de cómo se relaciona el calentamiento atmosférico en esta región con la variabilidad estacional de la precipitación observada en la cuenca alta del Mantaro.

En esta investigación, el objetivo principal es proponer una primera aproximación a una metodología, la cual, mediante la identificación de ciertas condiciones asociadas a la Radiación de Onda Larga Emergente medidas en la zona central del Océano Pacífico y del análisis de los procesos físicos y las dinámicas atmosféricas vinculadas con la generación de precipitaciones, pueda caracterizar los años con presencia de eventos extremos, como el evento La Niña, sobre la cuenca alta del Mantaro y, de esta manera, mejorar nuestra capacidad de pronóstico estacional en la cuenca alta del río Mantaro. Para ello, técnicas estadísticas como el Análisis de Correlación Lineal, el Análisis de Componentes Principales, etc. serán utilizadas para el estudio de la variabilidad y predictibilidad de la precipitación. Por otro lado, para obtener una mejor comprensión de la correlación entre estas variables, se realizará un análisis espacio temporal de las precipitaciones y de la Radiación de Onda Larga Emergente, así como un análisis de la circulación atmosférica relacionada con la generación de precipitaciones para los periodos lluviosos y secos.

Finalmente, la importancia de la presente investigación está sustentada en que la economía de muchos países de América del Sur, como el caso del Perú, se sustenta, en gran medida, en actividades como la agricultura, producción de energía eléctrica, etc. actividades que dependen de la disponibilidad del recurso hídrico, el cual se ve seriamente afectado por la ocurrencia de fenómenos extremos (Lagos et al., 2008). Por ello, regiones como la cuenca alta del río Mantaro en los Andes Centrales del Perú son 
altamente vulnerables a fenómenos meteorológicos extremos asociados a la variabilidad del clima; la cual se incrementará en los próximos años debido al cambio climático.

\section{Material y mètodos}

\section{Zona de estudio}

La cuenca del Mantaro, geográficamente, se encuentra ubicada entre los frentes occidental y oriental de los Andes centrales del Perú $\left(76.65^{\circ}-73.9^{\circ} \mathrm{W} ; 14.76^{\circ}-13.54^{\circ} \mathrm{S}\right)$, abarcando las regiones de Pasco, Junín, Huancavelica y Ayacucho, con una topografía cuya altitud varía entre 500 a 5300 m.s.n.m. debido a la presencia de la cadena montañosa de los Andes y un sistema de glaciares localizados en la parte central Este, con gran influencia en los procesos atmosféricos que se producen al interior de cuenca.

\section{Información climática.}

\section{Precipitación.}

La información pluviométrica, a nivel mensual y diaria, corresponde a las estaciones meteorológicas administradas por el Servicio Nacional de Meteorología e Hidrología (SENAMHI) y a la empresa ELECTROPERÚ, las cuales se encuentran ubicadas dentro del ámbito de la cuenca alta del río Mantaro. El periodo de registro de datos es de 1965-2015.

Tabla 1: Ubicación de estaciones meteorológicas en la cuenca alta del Río Mantaro.

\begin{tabular}{|c|c|c|c|c|c|c|c|}
\hline $\mathbf{N}^{\mathbf{o}}$ & Nombre & Tipo & Distrito & Provincia & Latitud & Longitud & $\begin{array}{l}\text { Altitud } \\
\text { (m.s.n.m) }\end{array}$ \\
\hline 1 & Cercapuquio & PLU & Chongos Alto & Huancayo & $12^{\circ} 22^{\prime} 49^{\prime \prime}$ & $75^{\circ} 18^{\prime} 09^{\prime \prime}$ & 4390 \\
\hline 2 & Cerro de Pasco & PLU & Chaupimarca & Yauli & -10.693 & -76.26 & 4391 \\
\hline 3 & Chilicocha & PLU & Acombambilla & Moya & $12^{\circ} 42^{\prime} 04^{\prime \prime}$ & $75^{\circ} 27^{\prime} 08^{\prime \prime}$ & 4275 \\
\hline 4 & Choclococha & PLU & Santa Ana & Castrovirreyna & $13^{\circ} 11^{\prime} 1^{\prime \prime}$ & $75^{\circ} 5^{\prime} 1^{\prime \prime}$ & 4201 \\
\hline 5 & Huancalpi & PLU & Vilca & Huancavelica & $12^{\circ} 32^{\prime} 22^{\prime \prime}$ & $75^{\circ} 14^{\prime} 12^{\prime \prime}$ & 3800 \\
\hline 6 & Huancavelica & PLU & Ascensión & Huancavelica & $\begin{array}{l}12^{\circ} 46^{\prime} \\
17.86^{\prime \prime}\end{array}$ & $75^{\circ} 0^{\prime} 44.52^{\prime \prime}$ & 3676 \\
\hline 7 & Hueghue & PLU & $\begin{array}{c}\text { Santa Barbara de } \\
\text { Carhuacayan }\end{array}$ & Yauli & $11^{\circ} 14^{\prime} 14^{\prime \prime}$ & $78^{\circ} 15^{\prime} 51^{\prime \prime}$ & 4175 \\
\hline 8 & Huichicocha & PLU & Chongos Alto & Huancayo & $12^{\circ} 33^{\prime} 42^{\prime \prime}$ & $75^{\circ} 31^{\prime} 44^{\prime \prime}$ & 4700 \\
\hline 9 & Junín & PLU & Junín & Junín & $11^{\circ} 8^{\prime} 35.8^{\prime \prime}$ & $75^{\circ} 59^{\prime} 19.6^{\prime \prime}$ & 4120 \\
\hline 10 & La Oroya & PLU & La Oroya & Yauli & $11^{\circ} 31^{\prime} 1^{\prime \prime}$ & $75^{\circ} 54^{\prime} 1^{\prime \prime}$ & 3750 \\
\hline 11 & Lircay & PLU & Lircay & Angares & $12^{\circ} 58^{\prime} 55^{\prime \prime}$ & $74^{\circ} 43^{\prime} 5.1^{\prime \prime}$ & 3150 \\
\hline 12 & Marcapomacocha & $\mathrm{CO}$ & Marcapomacocha & Yauli & $11^{\circ} 24^{\prime} 16^{\prime \prime}$ & $76^{\circ} 19^{\prime} 31^{\prime \prime}$ & 4479 \\
\hline 13 & Pachacayo & PLU & Canchayllo & Jauja & $11^{\circ} 49^{\prime} 18^{\prime \prime}$ & $75^{\circ} 43^{\prime} 09^{\prime \prime}$ & 3550 \\
\hline
\end{tabular}




\begin{tabular}{|c|c|c|c|c|c|c|c|}
\hline $\mathbf{N}^{\mathbf{0}}$ & Nombre & Tipo & Distrito & Provincia & Latitud & Longitud & $\begin{array}{c}\text { Altitud } \\
\text { (m.s.n.m) }\end{array}$ \\
\hline 14 & Palaco & PLU & Chongos Alto & Huancayo & $12^{\circ} 20^{\prime} 49^{\prime \prime}$ & $75^{\circ} 17^{\prime} 58^{\prime \prime}$ & 3850 \\
\hline 15 & Tambo de Sol & PLU & Parco & Jauja & $10^{\circ} 52^{\prime} 26^{\prime \prime}$ & $76^{\circ} 07^{\prime} 08^{\prime \prime}$ & 4100 \\
\hline 16 & Tarma & PLU & Tarma & Tarma & $11^{\circ} 23^{\prime} 49^{\prime \prime}$ & $75^{\circ} 41^{\prime} 25^{\prime \prime}$ & 3000 \\
\hline 17 & Telleria & PLU & Nahuimpuquio & Tayacaja & $12^{\circ} 22^{\prime} 30^{\prime \prime}$ & $76^{\circ} 07^{\prime} 16^{\prime \prime}$ & 3050 \\
\hline 18 & Upamayo & PLU & Upamayo & Junín & $10^{\circ} 55^{\prime} 19^{\prime \prime}$ & $76^{\circ} 16^{\prime} 24^{\prime \prime}$ & 4080 \\
\hline 19 & Vilca & PLU & Vilca & Huancavelica & $12^{\circ} 07^{\prime} 49^{\prime \prime}$ & $76^{\circ} 50^{\prime} 0^{\prime \prime}$ & 3815 \\
\hline 20 & Yantac & CO. & Marcapomacocha & Yauli & $11^{\circ} 20^{\prime} 0^{\prime \prime}$ & $76^{\circ} 24^{\prime} 0^{\prime \prime}$ & 4600 \\
\hline 21 & Yauli & PLU & Yauli & Yauli & $11^{\circ} 40^{\prime} 0^{\prime \prime}$ & $76^{\circ} 5^{\prime} 0^{\prime \prime}$ & 4141 \\
\hline 22 & Yauricocha & PLU & Alis & Yauyos & $11^{\circ} 57^{\prime} 20^{\prime \prime}$ & $75^{\circ} 64^{\prime} 24^{\prime \prime}$ & 4375 \\
\hline
\end{tabular}

\section{Resultados y discusiòn}

El análisis de las series temporales y espaciales de la precipitación total diaria para el total de estaciones pertenecientes a los dos Grupos de estaciones previamente clasificados se realizó para los periodos de diciembre a abril en los años identificados con eventos La Niña. Según los procedimientos descritos en la metodología, se procedió a la aplicación de la técnica estadística de Análisis de Componentes Principales, se eligió la matriz de correlaciones, los cuales explican la cantidad total de precipitación producida durante dichos eventos.

De la variabilidad de la precipitación a nivel diario durante los eventos La Niña, se analizaron el primer y segundo modo de variabilidad temporal (CP-1 y CP- 2) y los dos principales modos de variabilidad espacial durante los eventos La Niña 1998/99, 1999/00, $2007 / 08$ y $2010 / 11$.

Es importante tener en cuenta que valores más altos de correlación de una determinada estación con respecto al componente principal, sugieren un mayor grado de representación mediante dicho componente. Por ello, se procedió a agrupar estaciones por el signo de su correlación, permitiendo seleccionar las estaciones cuyo comportamiento está siendo reflejado por dicho componente principal (León Altuna, 2014). 
Evento La Niña 1998-1999.

De diciembre de 1998 a mayo de 1999, los dos principales componentes llegan a explicar 68.76 de la varianza (55.0 por ciento para el CP-1 y el 13.76 por ciento para el CP-2).

Los resultados del análisis espacial de las precipitaciones para el evento La Niña sugieren que existe una correlación positiva para todas las estaciones. Respecto al análisis del primer $\mathrm{CP}$, se puede observar que el periodo de mayor precipitación ocurrió durante los primeros días de diciembre de 1999 hasta los últimos días del mes de febrero de 1999. Por otro lado, del análisis de variabilidad de la precipitación a nivel diario (EOF-02), muestra una correlación positiva para la mayoría de estaciones ubicadas en la parte norte de la cuenca alta, mientras que la zona sur y centro muestran correlaciones negativas; por lo que se sugiere que los valores negativos del componente principal representan la precipitación diaria de la zona Sur de la cuenca, mientras que valores negativos representan valores de precipitación de la zona sur de la cuenca del Mantaro. Por otro lado, del análisis de la CP-2, durante el evento La Niña 1998/99, se observa un periodo muy lluvioso desde los primeros días del mes de enero, hasta inicios del mes de marzo, con pequeños periodos de lluvia de corta duración como en la segunda semana del mes de diciembre de 1998 o a mediados del mes de abril de 1999. Para las estaciones con correlaciones negativas se observa ligeras diferencias; el periodo lluvioso se extiende, empezando en los primeros días de enero de 1999 hasta mediados del mes de abril del mismo año.

\section{Variabilidad diaria de la precipitación durante eventos La Niña.}

\section{Evento La Niña 1998-1999.}

De diciembre de 1998 a mayo de 1999, los dos principales componentes llegan a explicar 68.76 de la varianza (55.0 por ciento para el CP-1 y el 13.76 por ciento para el CP-2).

Los resultados del análisis espacial de las precipitaciones para los eventos La Niña sugieren que existe una correlación positiva para todas las estaciones. Con respecto al 
análisis del primer componente principal, se puede observar que el período de mayor precipitación ocurrió durante los primeros días de diciembre de 1998 hasta los últimos días del mes de marzo de 1999. Por otro lado, el análisis de variabilidad de la precipitación a nivel diario (EOF-01), muestra una correlación alta para la mayoría de las estaciones de la cuenca alta.

Para el evento La Niña de diciembre de 1999 a mayo de 2000, los dos principales componentes llegan a explicar 59.7 de la varianza (45.4 por ciento para el CP-1 y el 14.3 por ciento para el CP-2). Para el evento La Niña de diciembre del 2007 a mayo de 2008, los dos principales componentes llegan a explicar 59.7 de la varianza (36.0 por ciento para el CP-1 y el 12.8 por ciento para el CP-2) y para el evento La Niña de diciembre del 2010 a mayo del 2011, los dos principales componentes llegan a explicar 59.7 de la varianza (41.2 por ciento para el CP-1 y el 11.88 por ciento para el CP-2). 


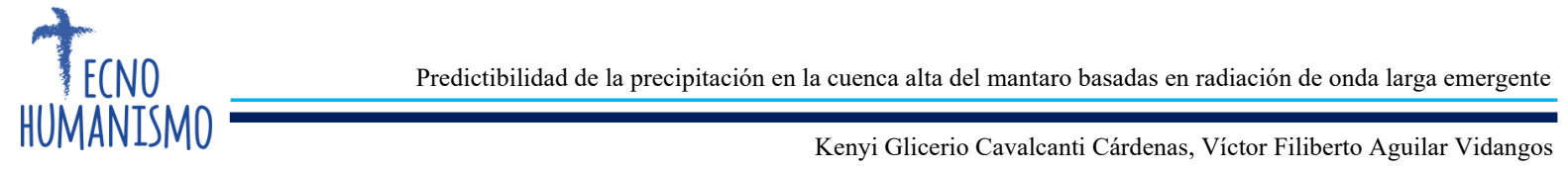

Figura 01.- Variación temporal y espacial de la precipitación diaria de EOF-1 durante el evento La Niña 1998/99, 1999/00, 2007/08, 2010/11
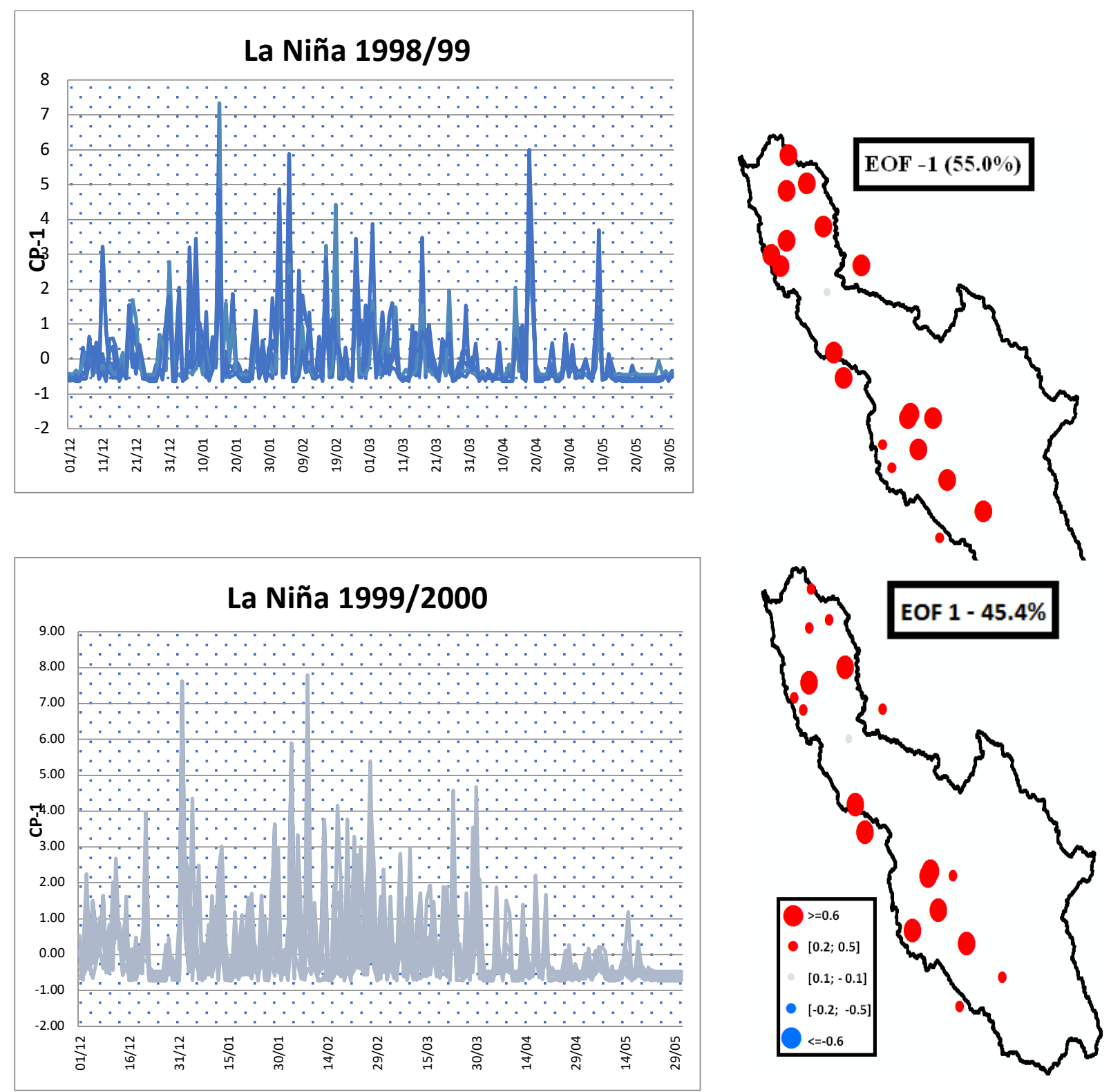

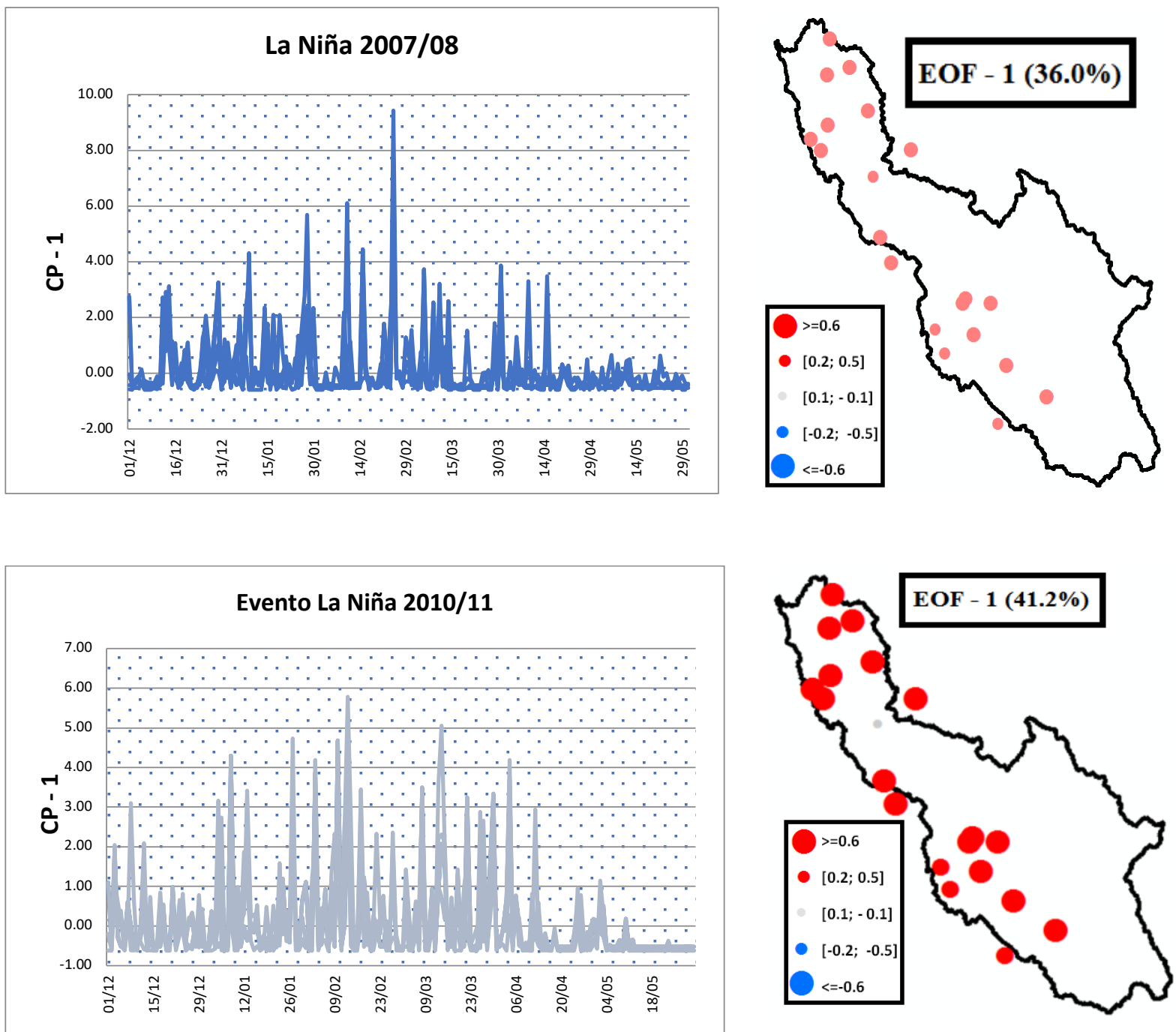

Relación entre el régimen de precipitaciones y la Temperatura Superficial del Mar.

Se presenta el análisis de la correlación entre las precipitaciones en la cuenca alta del Mantaro y las regiones de monitoreo El Niño, sobre la base de los estudios realizados por (Lagos et al., 2008). Para ello, se utilizó datos de precipitación a nivel mensual para las 22 estaciones analizadas, para el período 1965-2015, así como valores de temperatura superficial del mar en el océano Pacífico ecuatorial para cada una de las regiones de monitoreo: El Niño 1+2, Niño 3, Niño 3.4 y Niño 4.

Para el mes de noviembre, resalta la correlación positiva de las precipitaciones con todas las regiones El Niño, con valores estadísticamente significativos en algunas estaciones como Huichicocha y Yauricocha. Es importante señalar que un calentamiento en el Pacífico Occidental (Niño 4) se asocia a una mayor precipitación en la cuenca del Mantaro para este mes. Durante los meses de verano, la correlación entre la precipitación 
y la TSM es inversa, alcanzando mayores valores durante el mes de febrero; además se hace más significativa a medida que se toman valores de TSM de la región Niño 4 $\left(160^{\circ} \mathrm{E}-150^{\circ} \mathrm{W}, 5^{\circ} \mathrm{S}-5^{\circ} \mathrm{N}\right)$, donde dichos valores de correlación son mayores a los mostrados en otras regiones de monitoreo para la mayoría de las estaciones.

Estos resultados permiten apreciar que solo hay una correlación importante entre las TSM y las precipitaciones de la cuenca del Mantaro durante los meses de verano, especialmente febrero. Esta relación inversa, sugiere que anomalías positivas en el Pacífico central u occidental inhiben la precipitación en la parte central y sur de la cuenca del Mantaro, mientras que un enfriamiento en dicha región favorece la ocurrencia de precipitaciones. Estos resultados son consistentes con estudios previos (Yamina Silva Vidal, 2014), los cuales encontraron una correlación significativa entre las precipitaciones en la parte central de los Andes y la fase caliente del Índice de Oscilación del Sur, especialmente durante la temporada lluviosa. Esta correlación era particularmente fuerte para la zona central del Pacífico Tropical, lo cual sugería la influencia de la Temperatura Superfial del Mar sobre el régimen de precipitaciones.

En los resultados se muestra que las correlaciones en el mes de noviembre no muestran valores significativamente relevantes. Por otro lado, si bien no se muestran valores significativos para la mayoría de las estaciones durante este mes, se aprecia un patrón de comportamiento importante, en el cual un calentamiento en la región Central del Pacífico (Niño 4) se asocia a mayores precipitaciones en la cuenca alta del Mantaro, especialmente en la zona norte, mientras que un enfriamiento en el Pacífico Central se relaciona con menores precipitaciones sobre nuestra zona de estudio. Por otro lado, durante los meses de mayor precipitación se muestran valores de correlación negativa importantes con la región del Pacífico Occidental (Niño 4). 
Figura 2. Correlación entre las lluvias y la TSM en la región del Pacifico Oriental (Niño 1+2), Central (Niño 3, Niño 3.4) y Occidental (Niño 4) para el mes de febrero y noviembre.
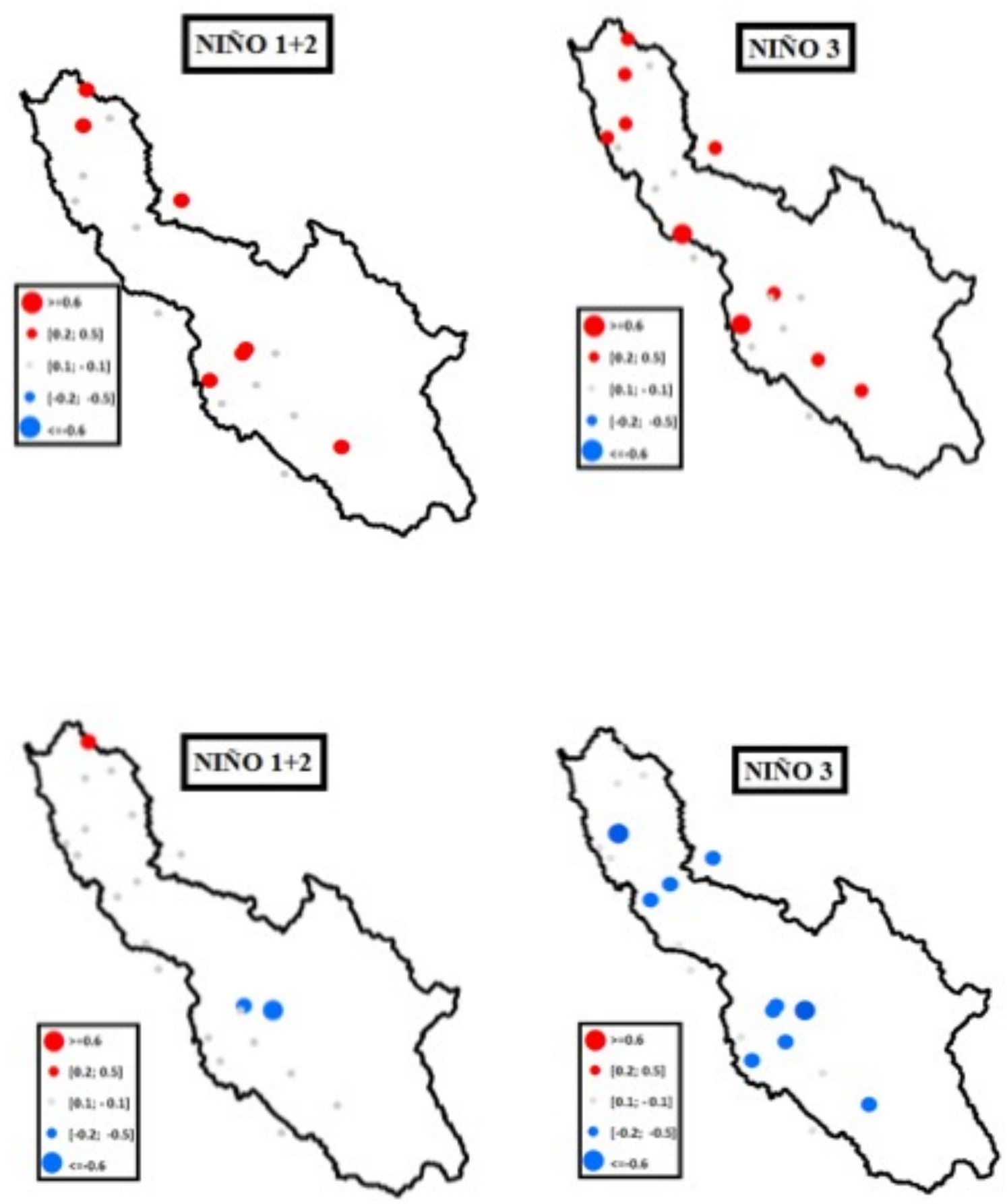

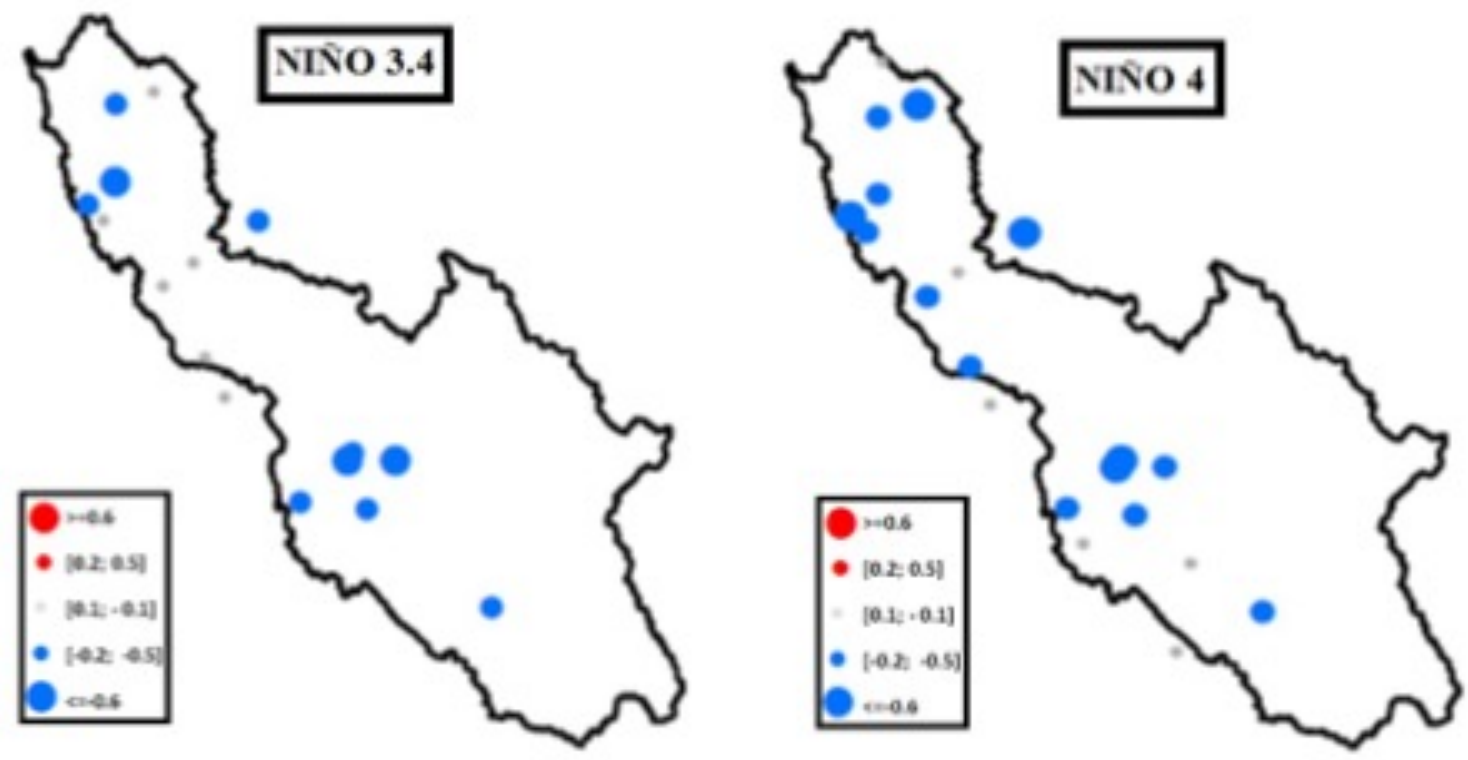

Adicionalmente se analizó las correlaciones de anomalías de OLR en el Pacífico Central con las anomalías de precipitación solo para aquellos años identificados con la presencia de eventos La Niña. En la tabla 2 se aprecia valores se correlación positivos y considerablemente más altos para los meses de mayor precipitación, especialmente en febrero, lo cual confirma que durante estos eventos los valores de OLR están vinculados a mayor presencia de nubes en el Pacífico y, mediante procesos de teleconexión, con mayores precipitaciones en la cuenca alta del Mantaro.

Tabla 2.- Correlación de anomalías de precipitación observada con anomalías de OLR en el Pacífico Central a nivel mensual durante evento La Niña.

\begin{tabular}{|l|c|c|c|c|c|c|}
\hline & OCT & NOV & DIC & ENE & FEB & MAR \\
\hline Cercapuquio & 0.06 & 0.09 & -0.09 & 0.16 & 0.38 & 0.56 \\
\hline Cerro de Pasco & -0.40 & -0.11 & -0.07 & 0.37 & 0.27 & -0.18 \\
\hline Chilicocha & 0.33 & 0.47 & 0.58 & -0.14 & 0.55 & 0.57 \\
\hline Choclococha & 0.03 & 0.50 & 0.52 & -0.10 & -0.15 & 0.05 \\
\hline Huancalpi & -0.01 & 0.02 & 0.11 & 0.21 & 0.41 & -0.16 \\
\hline Huancavelica & -0.09 & 0.25 & 0.80 & 0.04 & 0.49 & -0.15 \\
\hline Huegue & -0.15 & -0.17 & 0.40 & 0.21 & 0.68 & 0.21 \\
\hline Huichicocha & 0.37 & -0.22 & 0.09 & 0.41 & 0.52 & 0.34 \\
\hline La Oroya & -0.30 & 0.22 & 0.19 & 0.59 & 0.24 & 0.21 \\
\hline Lircay & 0.03 & 0.21 & 0.60 & 0.15 & 0.53 & -0.12 \\
\hline
\end{tabular}




\begin{tabular}{|l|c|c|c|c|c|c|} 
Marcapomacocha & 0.00 & 0.12 & -0.11 & 0.29 & 0.45 & -0.16 \\
\hline Pachacayo & 0.34 & 0.21 & -0.09 & 0.03 & 0.21 & 0.29 \\
\hline Palaco & 0.18 & 0.13 & 0.38 & 0.14 & 0.75 & 0.51 \\
\hline Tambo de Sol. & 0.34 & -0.28 & 0.14 & -0.19 & 0.19 & 0.54 \\
\hline Tarma & 0.55 & 0.38 & 0.58 & 0.74 & 0.19 & -0.10 \\
\hline Telleria & 0.52 & -0.14 & 0.70 & -0.21 & 0.38 & 0.56 \\
\hline Upamayo & 0.12 & -0.20 & -0.09 & -0.75 & -0.27 & 0.31 \\
\hline Vilca & 0.06 & -0.31 & 0.33 & 0.11 & -0.25 & 0.14 \\
\hline Yantac & 0.06 & -0.02 & 0.68 & 0.35 & 0.32 & -0.03 \\
\hline Yauli & 0.26 & -0.09 & 0.17 & 0.45 & 0.47 & -0.07 \\
\hline Yauricocha & -0.09 & -0.29 & 0.08 & -0.42 & 0.53 & -0.57 \\
\hline
\end{tabular}

\section{Análisis del pronóstico de precipitaciones sobre la base de OLR.}

El análisis de los valores de OLR mensuales para el periodo 1974 - 2013 muestra un comportamiento diferenciado de los demás años con valores de OLR por encima de lo normal para años identificados eventos La Niña.

En la figura 3 se resalta las condiciones presentadas en el evento El Niño 1997/98 con altos valores de anomalías negativas de OLR en todo el Pacífico central y oriental, el cual se extiende hasta el mes de julio del año siguiente. Asimismo, se aprecian anomalías positivas de los valores de OLR en el Pacífico central y occidental, el cual está relacionado con un enfriamiento en la región El Niño 4, así como una disminución de presión sobre Indonesia y un aumento en la actividad convectiva en esta región.

Figura 3: Variación temporal y longitudinal de anomalías de la radiación de onda larga (OLR) promedio para la región comprendida entre 5N-5S. El intervalo entre contornos es $10 \mathrm{Wm}-2$ para el periodo Jul (2005) a Abril (2015). Los contornos discontinuos indican anomalías OLR negativas. Las anomalías son salidas desde 19812010 base período mensual medio. 


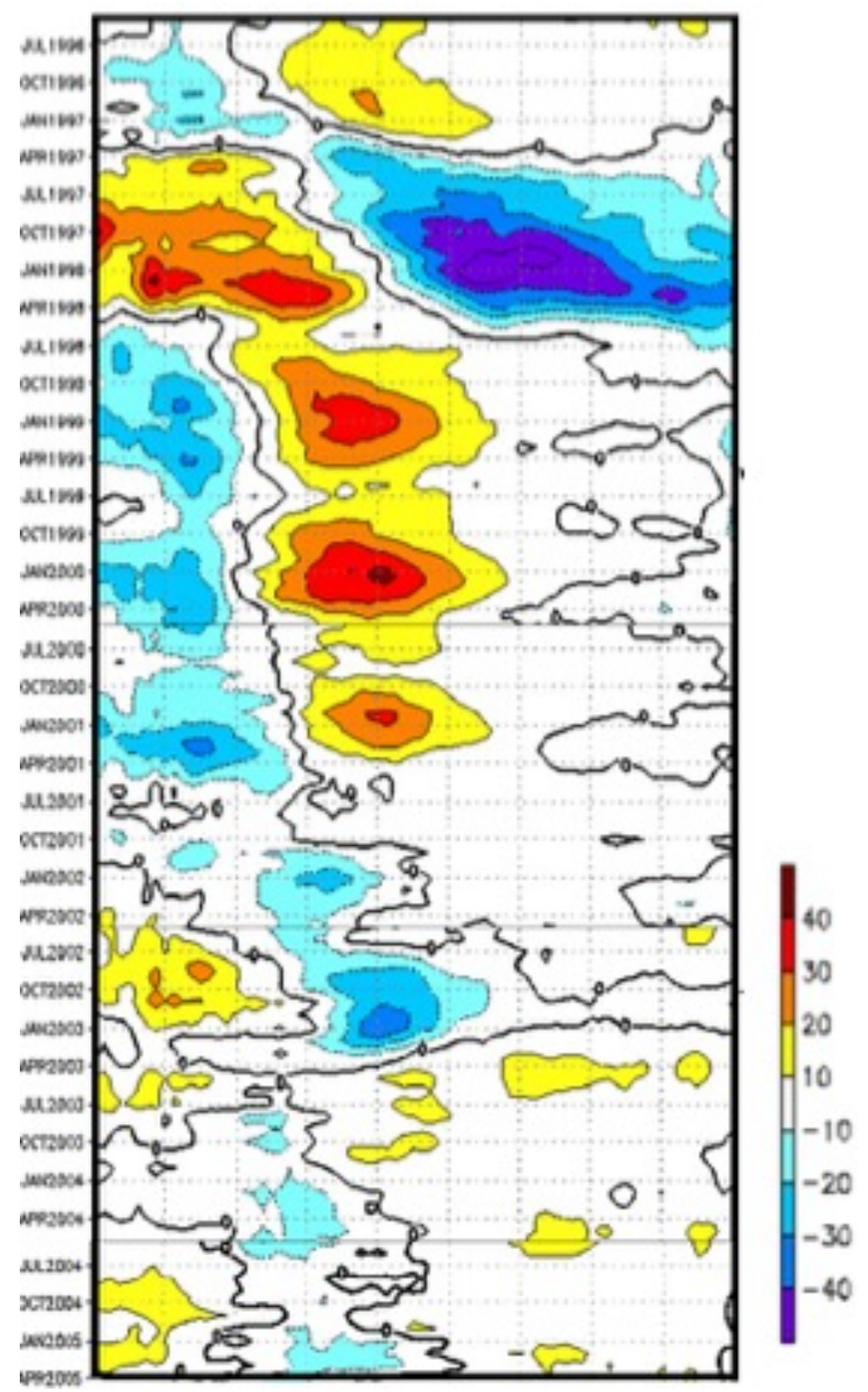

A partir de julio de 1998 aparecen fuertes anomalías positivas en la región conocida como El Niño 4, las cuales se intensifican durante los meses de diciembre a marzo del 2000, años donde la precipitación en la cuenca alta del Mantaro alcanza sus mayores valores, llegando a valores promedio de $870 \mathrm{~mm} /$ año para las estaciones ubicadas al sur y un valor promedio de $952 \mathrm{~mm} /$ año para las estaciones ubicadas al norte de la cuenca alta del Mantaro. Asimismo, se muestra la presencia de anomalías positivas desde octubre del año 2005, llegando a sus mayores valores durante los meses de lluvia del 2006, lo cual se ve reflejado en un aumento muy importante de las precipitaciones en todas las estaciones de la cuenca del Mantaro, luego de un período con déficit de precipitaciones observado durante el año 2005, lo cual podría ser explicado por condiciones promedio de OLR, es decir, con anomalías cercanas a cero, condiciones semejantes a la presentadas durante el 2012. Por otro lado, se observa que durante los periodos con anomalías 
negativas de OLR se presentan periodos más largos de días secos consecutivos (índice CDD) como es el caso de los años 2003 y 2010 donde se presentan periodos de hasta 60 días secos consecutivos.

Por otro lado, para un mejor análisis de la correlación entre los valores de OLR en el Pacífico central, se promediaron los valores diarios registrados dentro de la zona conocida como Niño $4\left(160^{\circ} \mathrm{W}-150^{\circ} \mathrm{E} ; 5^{\circ} \mathrm{S}-5^{\circ} \mathrm{N}\right)$. A continuación, se presentan los valores diarios de OLR promedio, desde el mes de marzo del año anterior (Año -1) hasta el mes de enero del año identificado con la presencia del evento La Niña. Del análisis de los valores diarios de OLR previos a los meses de mayor precipitación en la cuenca alta del Mantaro, se puede identificar un patrón que caracteriza estos meses. En los meses previos a los de mayor precipitación, se registraron valores desusadamente altos de OLR en la región El Niño 4, por lo cual se adaptó el Índice OLR-La Niña (OLNI) definido por (Chiodi \& Harrison, 2015), con la finalidad de ver la capacidad de pronóstico de grandes valores de precipitaciones en la cuenca del Mantaro. Se muestran valores muy altos a partir del mes de junio del año -1, los que, en su mayoría sobrepasan el valor de $270 \mathrm{~W} / \mathrm{m} 2$ (valor igual al percentil 90), siendo la principal característica distintiva de los meses previos a un evento La Niña. Es importante mencionar que el número de días contabilizados es a partir del 1 de marzo del año previo al identificado con un evento La Niña, y finaliza el 31 de diciembre de ese mismo año (año -1). Dicho período se ha identificado como el correspondiente entre el inicio y el desarrollo del evento La Niña, el cual permite analizar la capacidad de predicción meses previos al período de mayor precipitación en la cuenca alta del Mantaro.

Como se ha detallado en la metodología, el promedio diario de los valores de OLR se realizó sobre la región de El Niño $4\left[160^{\circ} \mathrm{E}, 150^{\circ} \mathrm{W}\right.$ y $\left.-5^{\circ} \mathrm{S} 5^{\circ} \mathrm{N}\right]$ por ser la que mejor correlación muestra con la cuenca alta del Mantaro. Por último, se estableció un valor de

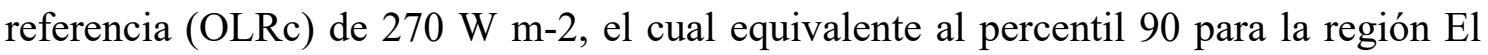
Niño 4. La figura 4 muestra la evolución de los valores diarios de OLR en la región El Niño 4, en los cuales se aprecia que, durante los primeros días del mes de agosto, los valores de OLR superan el valor límite de $270 \mathrm{~W} / \mathrm{m} 2$, manteniendo este comportamiento durante los meses de mayor precipitación. 
Figura 4: Valores diarios promediados de OLR para la región del Pacífico Central $\left(160^{\circ} \mathrm{W}-150^{\circ} \mathrm{E} ; 5^{\circ} \mathrm{S}-5^{\circ} \mathrm{N}\right)$ desde el mes de marzo del año anterior (año -1$)$ hasta el mes de enero (año 0) del evento La Niña 1998/99.
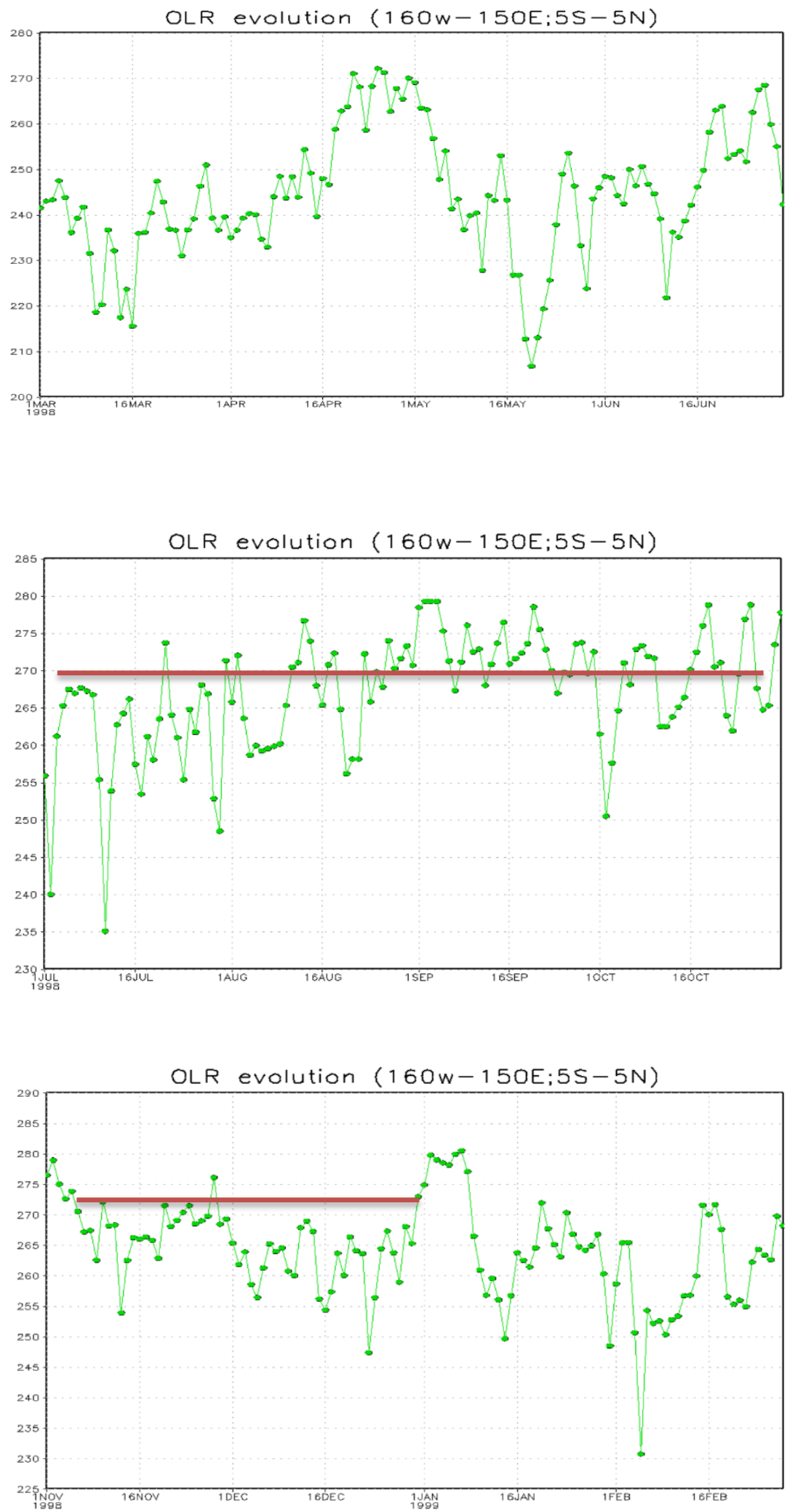
Posteriormente, se procedió a calcular el Índice OLR- La Niña. Los resultados se muestran en la figura 5 donde se destacan los eventos La Niña extraordinarios (1973/74, 1975/76, 1988/89) y La Niña moderados (1998/99, 1999/00, 2007/08 y 2010/11), diferenciándose de los años subsiguientes en aproximadamente la mitad del valor de la desviación estándar (el valor de OLNI para el año 1988 fue 113, mientras que el siguiente año alcanzó un valor de 31).

El Índice OLR La Niña (OLNI) mediante el cual se lograron identificar tres años donde el número el índice OLR La Niña supera el valor de 80 días, cabe mencionar que ningún otro año logra exceder esta cantidad para el período de análisis de precipitaciones diaria (1998 - 2015).

Figura 5: Variación temporal del Índice OLR La Niña (OLNI).

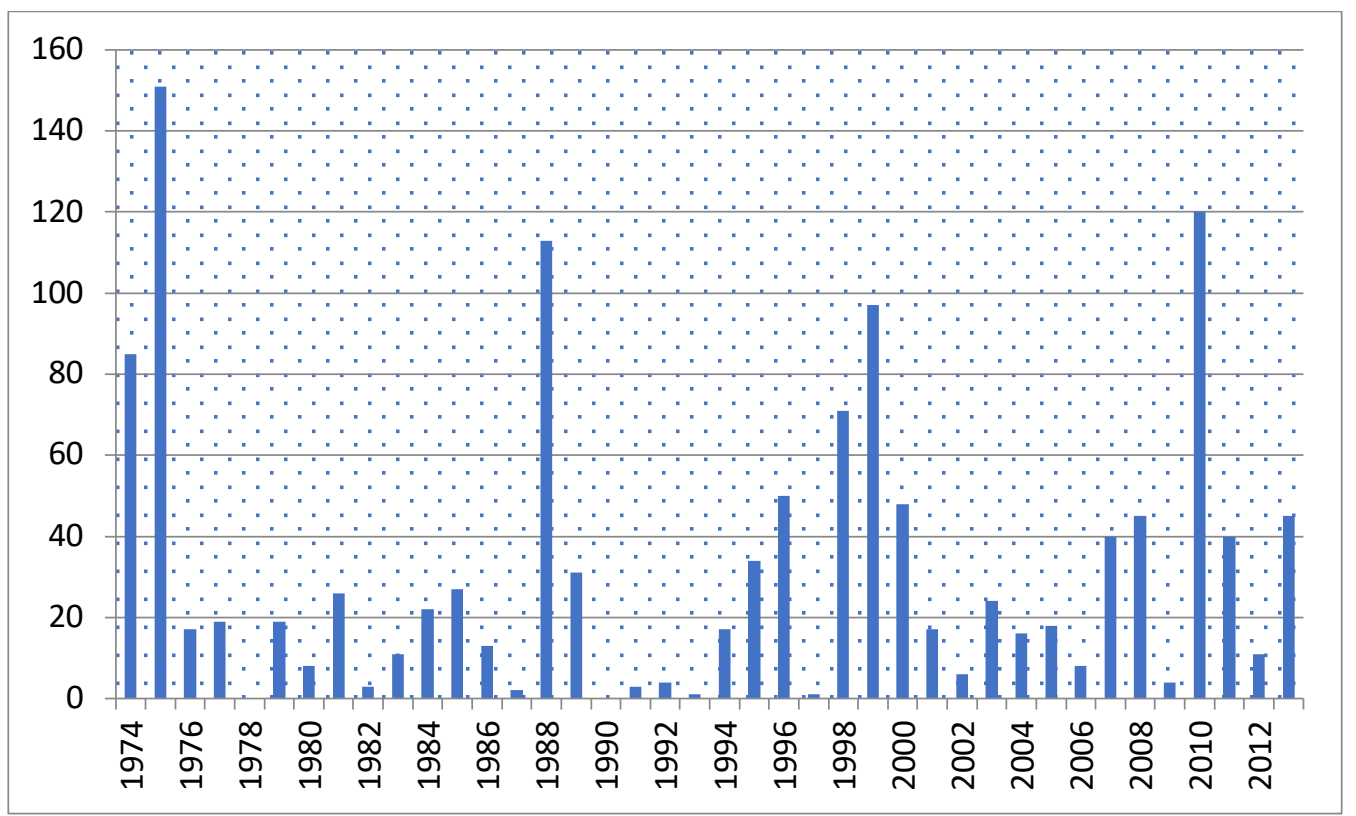

\section{Conclusiones}

Los resultados muestran que la cuenca alta del Mantaro presenta una variabilidad hidrológica fuertemente modulada por cambios en el Pacífico Central, conocido como región Niño 4, por cuanto, un enfriamiento en dicha región se correlaciona con mayores precipitaciones y mayores caudales en la cuenca del Mantaro; por otro lado, altos valores de anomalías positivas de OLR están relaciones con un aumento de las precipitaciones, especialmente durante eventos La Niña. Asimismo, se observa que un enfriamiento del Pacífico Central, durante los meses de mayor precipitación, está vinculado con un 
incremento de la precipitación total anual, frecuencia de días húmedos, y días con precipitaciones extremas. Por otro lado, los resultados muestran mayores valores de correlaciones con caudales observados, en comparación a los resultados obtenidos con la variable precipitación, lo cual podría deberse a que éstas últimas ofrecen una mejor representación de la hidrología de la cuenca al ser representativas de una determinada área a diferencia de los valores puntuales de lluvia proporcionados por las estaciones,

Se muestra una perspectiva distinta a la comúnmente usada para analizar la predictibilidad de precipitaciones y caudales en una determinada región sobre la base del comportamiento de la Radiación de Onda Larga Emergente (OLR) en el Pacífico Central. Los resultados muestran que un enfriamiento anómalo de dicha región del Pacífico Tropical favorece la presencia de mayores valores de precipitación en la cuenca alta del Mantaro; empero, este comportamiento se caracteriza por una cantidad desusadamente alta de días con altos valores de OLR $(270 \mathrm{w} / \mathrm{m} 2)$ en meses previos a la ocurrencia de grandes periodos de precipitación y a la presencia de eventos La Niña.

Asimismo, mediante la aplicación del índice OLR se identificaron claramente seis eventos La Niña en el período 1974-2015 en los cuales, los regímenes de precipitación registrados en la cuenca del Mantaro son considerablemente superiores a los del promedio, ofreciendo una mayor facilidad y precisión en la identificación de dichos eventos, en contraste con la comúnmente utilizada técnica basada en valores de Temperatura Superficial del Mar.

\section{Referencias bibliográficas}

Chiodi, A. M., \& Harrison, D. E. (2015). Global seasonal precipitation anomalies robustly associated with El Niño and La Niña events - an OLR perspective. Journal of Climate, 28(15), 6133-6159. https://doi.org/10.1175/JCLI-D-1400387.1

Lagos, P., Silva, Y., Nickl, E., \& Mosquera, K. (2008). El Nin $\pm_{0}$ - Related precipitation variability in Perú. Advances in Geosciences, 14(3), 231-237.

https://doi.org/10.5194/adgeo-14-231-2008

León Altuna, K. B. (2014). Análisis Espacio-Temporal De Las Precipitaciones $Y$ Caudales Durante Los Eventos El Niño (1982-83 Y 1997-98) En La Costa Norte Peruana. 1-146.

Segura, H., Espinoza, J. C., Junquas, C., \& Takahashi, K. (2016). Evidencing decadal 
and interdecadal hydroclimatic variability over the Central Andes. Environmental Research Letters, 11(9), 1-8. https://doi.org/10.1088/1748-9326/11/9/094016

Silva, Y., Takahashi, K., \& Chávez, R. (2008). Dry and wet rainy seasons in the Mantaro river basin (Central Peruvian Andes). Advances in Geosciences, 14, 261264. https://doi.org/10.5194/adgeo-14-261-2008

Sulca, J., Vuille, M., Silva, Y., \& Takahashi, K. (2016). Teleconnections between the peruvian central andes and northeast Brazil during extreme rainfall events in austral summer. Journal of Hydrometeorology, 17(2), 499-515. https://doi.org/10.1175/JHM-D-15-0034.1

Yamina Silva Vidal, P. D. (2014). Correlación entre las lluvias y la TSM en el Pacífico occidental (Niño 4) para los meses de noviembre y febrero Artículo de Divulgación. Niño 4. 